\title{
Precision and accuracy of repeat ultrasound image acquisition and analysis of the cross-sectional areas of the equine flexor tendons of the forelimbs for follow-up assessments
}

\author{
Kaid Kojah', Mandy Vogel ${ }^{2}$ and Jenny Hagen ${ }^{7}$ \\ 1 Institute of Veterinary Anatomy, Histology and Embryology, Faculty of Veterinary Medicine, Leipzig University, Germany \\ 2 LIFE - Research Center for Civilization Diseases, Hospital for Children and Adolescents, Leipzig University, Germany
}

\begin{abstract}
Summary: Ultrasound imaging of the distal limb enables veterinarians to determine morphological characteristics like the size and degree of changes in tissue conformation of the flexor tendons. To date, no study has validated the accuracy and precision of repeat follow-up ultrasound examinations for quantitative measurements of the cross-sectional area (CSA) of both flexor tendons. So this study was perforemed evaluate the accuracy and precision of ultrasound image acquisition and analysis to evaluate the cross-sectional areas of the equine deep and superficial digital flexor tendons of the forelimbs. In 10 consecutively obtained transverse images the CSA of both flexor tendons at 3 levels was examined in 5 horses by one operator. To examine the agreement of repeat image analysis, the CSA of the SDFT and DDFT at 3 levels on the left forelimb of 12 sound warmblood horses was repeatedly measured 10 times in the obtained ultrasound images by the same experienced operator. For all examinations the overall concordance correlation coefficient (OCCC) was calculated. The OCCC of the repeated image acquisition at the SDFT was very high at all levels $(\geq 0.98-\geq 0.99)$ and at the DDFT, a substantial or modest agreement for level 2A (0.98) and 2B (0.94) and a poorer agreement at level 1B (0.84) was shown. Repeat image analysis of the CSA showed an extremely high agreement between all measurements of the cross-sectional area at all levels of both tendons $(\geq 0.99)$. The results show that the acquisition and analysis of transverse ultrasound images of the SDFT and the DDFT to measure the cross-sectional areas at different levels can be stated as a technique to perform quantitative examinations with high precision and accuracy for scientific purpose or as follow-up examinations of clinical cases.
\end{abstract}

Keywords: horse, ultrasound imaging, digital superficial flexor tendon, deep digital flexor tendon, forelimb, cross-sectional area, concordance correlation coefficient

Citation: Kojah K., Vogel M., Hagen J. (2017) Precision and accuracy of repeat ultrasound image acquisition and analysis of the crosssectional areas of the equine flexor tendons of the forelimbs for follow-up assessments. Pferdeheilkunde 33, 320-328; DOI 10.21836/ PEM20170401

Correspondence: Dr. Jenny Hagen, Institute of Veterinary Anatomy, Histology and Embryology, Faculty of Veterinary Medicine, Leipzig University, An den Tierkliniken 43, 04103 Leipzig, Germany; e-mail: hagen@vetmed.uni-leipzig.de

\section{Introduction}

Ultrasound examination of the equine distal limb to detect tendon lesions has become a commonly applied method of diagnostics in equine practice (Marr et al. 1993, Denoix 1994, Dowling et al. 2000). Ultrasound imaging of the distal limb enables veterinarians to determine morphological characteristics like the size and degree of changes in tissue conformation of the flexor tendons (Hauser 1986, Henry et al. 1986, van Schie and Bakker 2000). To a certain extent, it can allow veterinarians to monitor the healing of lesions and to determine a prognosis for reintroducing the horse to training. Moreover, quantitative ultrasound examinations are used in a scientific context to assess the impact of various factors, such as training, growth, or breed, on the morphology of the flexor tendons (Gillis et al. 1995, Agut et al. 2009, Avella et al. 2009, Boehart et al. 2010). For this purpose, follow-up examinations of the same horse are performed to compare the tendon properties between treatments or during different phases of recovery. Commonly assessed parameters include the echogenicity, size (cross-sectional area, CSA), differentiation from surrounding structures, and shape measured at different levels of the digital flexor tendons between the carpus and the metacarpophalangeal joint (Gillis et al. 1995b, Wood et al.
1993, Smith et al. 1994). In particular, the echogenicity, describing alterations of the internal architecture of the tendons, and the increase in the cross-sectional areas are evaluated. These properties of tendons measured in ultrasound images are compared to adjacent structures, the contralateral limb, or under different conditions for clinical and scientific purpose (Nicoll et al. 1992, Tsukiyama et al. 1996). Despite technical improvement of ultrasound equipment, this method of diagnostics automatically implies that image acquisition and analyses, when done quantitatively, are both affected by the subjective view and experience of the person performing the examination, and therefore may not be ideal when comparability is important (Smith et al. 1994). High reproducibility of image acquisition and analyses is crucial for accurate diagnosis and detection of subtle changes in follow-up examinations. Objective criteria would be useful to estimate the accuracy and precision of repeat ultrasound examinations. (Pickersgill et al. 2001).

Therefore, certain standards have been developed to increase the validity and accuracy of ultrasound imaging with regard to the examination of the equine digital flexor tendons (Denoix 1994, Miles et al. 1996, van Schie et al. 1998, Padaliya et al. 2015). Studies have been carried out to determine quan- 
titative methods of evaluation such as grey scale analysis, including mean grey scale level or mean echogenicity (van Schie et al. 2000, van Schie and Bakker 2000). Studies considering the CSA mainly focused on the establishment of reference values and on the evaluation of the inter-operator or inter-equipment variability of image acquisition and analysis (Smith et al. 1994, Pickersgill et al. 2001, Vilar et al. 2011).

Thus far, no study has validated the accuracy and precision of repeat follow-up ultrasound examinations for quantitative measurements of the CSA of both flexor tendons of the left forelimb by one operator - an option to monitor changes of this parameter for different conditions in equine practice and research. Therefore, the aim of this study was to examine the following hypotheses by calculating the overall concordance correlation coefficient [OCCC], overall accuracy [OACC], and overall precision [OPREC]:

- With the given setup (as described in the method section), an experienced operator can acquire highly consistent images (OCCC, OACC, and OPREC substantial or perfect) to calculate the cross-sectional area of the superficial flexor tendon (SDFT) and deep digital flexor tendon (DDFT) at three different levels.

- With the given setup and software, an experienced operator can achieve highly consistent image analysis (OCCC, OACC, and OPREC substantial or perfect) of the cross-sectional area of the SDFT and DDFT at three different levels.

- The consistency in image acquisition and analysis of the cross-sectional area of the SDFT and the DDFT are high enough to detect subtle changes in the size of the tendons during repeat measurements under field conditions.

\section{Material and Methods}

Animals

A randomly selected cohort of 17 clinically sound warmblood horses $(147-168 \mathrm{~cm} ; 420-580 \mathrm{~kg}, 6-12$ years, 8 male and 9 female) was selected from a larger population of horses resident in a riding school. All horses were used to being handled, calm, and did not have severe irregularities of limb or hoof conformations. They were regularly trimmed or shod, and were housed, fed, and managed in a similar way. The animals experienced a similar level of exercise, approximately 10-15 h per week. Before any investigation, the horses independently underwent clinical, orthopaedic, radiological, and sonographic examination by 2 veterinarians who had more than 5 years' experience in equine practice and lameness diagnostics. Orthopaedic examination included inspection, palpation, and a lameness examination on hard and soft surface. Separate $90^{\circ}$ and $0^{\circ}$ radiographs of the toe and the fetlock were acquired and sonographic examinations of the soft tissue were performed to exclude pathologies of the distal limb. Based on this examination, horses with orthopaedic disorders or other diseases were excluded.

\section{Study Design}

Firstly, the reproducibility of the ultrasound image acquisition was evaluated. Therefore, in 5 horses 10 transverse images of the SDFT and DDFT at 3 levels were consecutively obtained by one operator, experienced in ultrasound examination. The ten images from one horse were assessed consecutively in one session. The probe was completely removed and the horses were moved between examinations. Subsequently, the CSAs were measured once in each of the obtained images.

For the ultrasound image acquisition, horses were standing square on a nonslip floor. Image acquisition was performed on the forelimbs of the horses without sedation. The palmar aspect of the metacarpal region was clipped and then scrubbed with alcohol. Echolucent gel ${ }^{\prime}$ was applied prior to and during the examination. With the probe contacting the most palmar aspect of the metacarpal region, perpendicular to its palmar surface, transverse images of the SDFT and DDFT were obtained at a predetermined level. For the examinations, a portable ultrasound unit ${ }^{2}$ and a variable frequency linear transducer ${ }^{3}(6.0-11 \mathrm{MHz}$; Width: $40 \mathrm{~mm})$, set at $8.5 \mathrm{MHz}$ and a field depth of $4 \mathrm{~cm}$ with a stand-off pad to ensure optimal coupling, were used. Each image was assessed $8 \mathrm{~cm}$ (zone 1B), $12 \mathrm{~cm}$ (zone 2A) and $16 \mathrm{~cm}$ (zone 2B) distal of the accessory carpal bone (Padaliya et al. 2015) (Figure 1), thus splitting the tendon convolute into three parts to obtain a good overview of the SDFT and DDFT throughout both their ranges where most pathologies are likely to occur, in particular at the SDFT. The DDFT is mainly affected by disorders located distally of the fetlock, however this region was not a focus of the present study and should be part of further examinations (Denoix 1994, Williams et al. 2001, Lam et al. 2007, Ely et al. 2009). The probe was manipulated in all planes to obtain an optimal image showing the widest cross-sectional area possible of each tendon at the specific level. Consistent perpendicular and palmar probe placement was ensured. Between image acquisitions the probe was removed and then replaced in the same position. Focus, brightness contrast, and gain where not modified throughout this study and thus remained the same for all acquired images to ensure comparability (Agut et al. 2009). To guarantee that the same level was chosen for each examination, a standard measuring tape was utilised before each image was acquired.

Secondly, part the reproducibility of the image analysis was examined. Therefore, the cross-sectional areas of the SDFT and DDFT were repeatedly measured 10 times in one image of each level on the left forelimb of 12 horses by the same experienced operator. All 10 measurements for one horse were performed consecutively at the same time.

For image analysis a still, representative image of the SDFT and the DDFT of each level was saved when allowing the accurate identification and tracking of the outer margin of both flexor tendons. The operator's name, the name of the horse, the examined limb (left, right), and the level of the examination (1B, 2A, 2B) were added to the recorded and stored image for identification during subsequent analyses. All images were stored on a USB stick and used for further analysis to calculate the cross-sectional areas of each tendon and at each level. All obtained images were saved as jpeg files and analysed using the open source image processing software ImageJ ${ }^{4}$. The CSA (cross-sectional area) of the SDFT and the DDFT was measured by outlining the structures in question with a cursor and then calculating the encompassed area. 


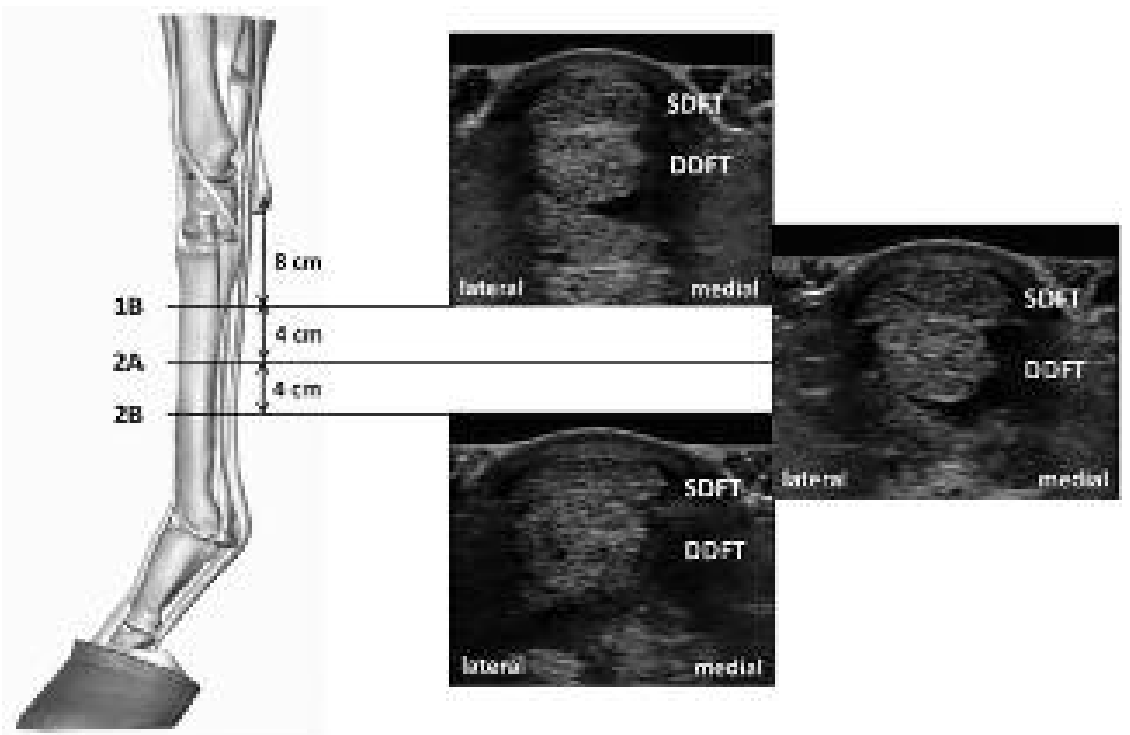

Fig. 1 Ultrasound examination to obtain transverse images of the SDFT and the DDFT of the forelimb at level $1 \mathrm{~B}, 2 \mathrm{~A}$, and $2 \mathrm{~B}$ (created and published with permission of Effigos "Hoof Explorer" www.hoofexplorer.com).

This was performed 10 times per image to compare the reproducibility of values produced with this software and by one experienced operator.

\section{Statistical Analysis}

Statistical analysis was carried out with the software ${ }^{5}$. Mean and standard deviations were calculated for the cross-sectional areas of the SDFT and DDFT at each level. In a validation process, the reproducibility of the data collection and quantitative measurements for analysis are of interest. The authors chose the overall concordance correlation coefficient (OCCC) to fully assess the desired reproducibility characteristics of image acquisition and analyses. The concordance correlation coefficient $c$ (CCC) evaluates the agreement between two values from the same sample by measuring the variation from the $45^{\circ}$ line through the origin (the concordance line). Lin's concordance correlation coefficient is stated to be the best measure of agreement for measuring the same continuous variable to evaluate reproducibility (Lin 1989, McBride 2005). First, it addresses the precision and accuracy indices as components of the OCCC. Second, it clarifies that the OCCC is the weighted average of pairwise CCCs. The OCCC is equivalent to the generalised CCC when the squared distance function is used (McBride 2005). In this study the overall CCC (OCCC) in terms of the inter-measurement variability for assessing agreement among multiple, repeat ultrasound image acquisition and analysis of the cross-sectional areas at different levels of the SDFT and the DDFT was applied. Additionally, the measures of overall accuracy (OACC) and overall precision (OPREC) are presented for the stated parameters. Accuracy is a measure of statistical bias describing the systematic errors as the weighted average of a measurement s sensitivity and specificity. OPREC of a measurement, related to reproducibility and repeatability, is the degree to which repeated data collection or measurements under the same conditions show the same results. McBride (2005) suggests the following descriptive scale for values of the concordance correlation coefficient for continuous variables: Value of OCCC strength of agreement $<0.90=$ poor, $0.90-0.95=$ moderate, $0.95-0.99=$ substantial,$>0.99=$ almost perfect (McBride 2005).

\section{Results}

All horses showed no evidence of SDFT or DDFT tendinitis or lesions $(n=17)$. Distinct inter-individual variations occurred for the size of the cross-sectional areas of the SDFT and DDFT ( $n=17$ horses) as shown by presenting the minimum, maximum and standard deviation (SD) for this parameter in Table 1.

Image acquisition - Reproducibility of assessing ultrasound images to evaluate the cross-sectional area of the digital flexor tendons.

Table 2 lists the mean and standard deviation (SD) of the cross-sectional areas $\left(\mathrm{mm}^{2}\right)$ of 10 repeat ultrasound assessments at each of the three levels (B1, A2, B2) of the SDFT and DDFT at the distal forelimbs of 5 horses. It shows that the SD

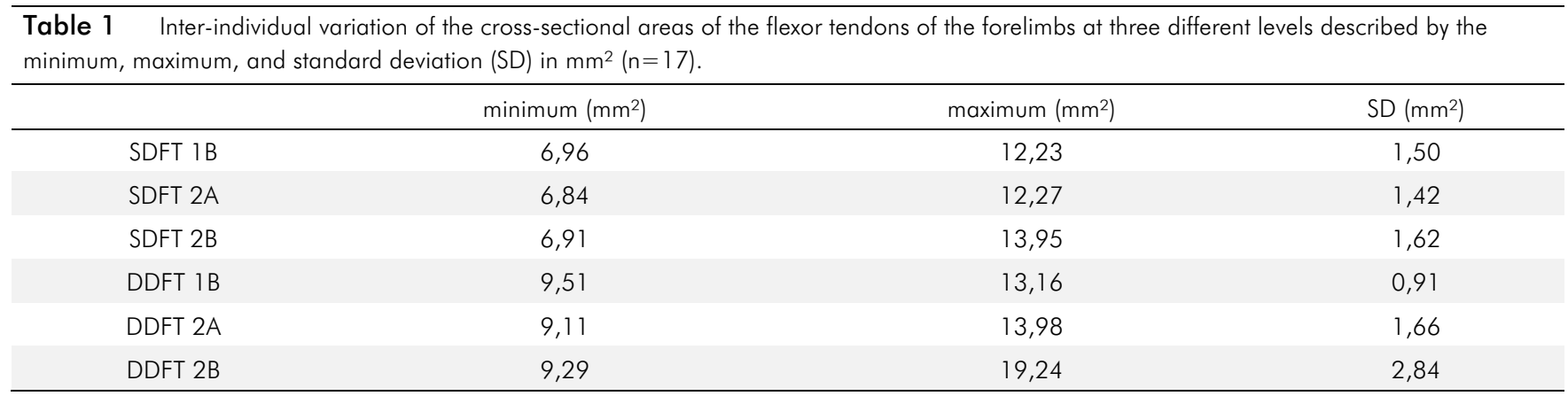


for repeat ultrasound imaging of the SDFT to evaluate the cross-sectional area is quite low $\left(0.07-0.29 \mathrm{~mm}^{2}\right)$. Differences in the dimensions of the SD are observed between the 5 horses. Similar results occurred for repeat ultrasound imaging of the DDFT. Here, values for SD between 0.10 and $0.28 \mathrm{~mm}^{2}$ occur.
Table 3 shows the statistical measures OCCC [overall concordance correlation coefficient], OACC [overall accuracy], and OPREC [overall precision] describing the reproducibility of the 10 ultrasound examinations to assess the cross-sectional area of the SDFT and the DDFT at three different levels $(1 \mathrm{~B}, 2 \mathrm{~A}, 2 \mathrm{~B})$ in 5 horses in vivo. In general, the given experi-

Table 2 Mean and standard deviation (SD) of the cross-sectional area $\left(\mathrm{mm}^{2}\right)$ of 10 ultrasound examinations at three levels (1B,2A, 2B) of the SDFT and DDFT $(n=5)$.

\begin{tabular}{ccccccc}
\hline Horse & SDFT 1B & SDFT 2A & SDFT 2B & DDFT 1B & DDFT 2A & DDFT 2B \\
\hline 1 & $9.27 \pm 0.16$ & $10.57 \pm 0.25$ & $9.67 \pm 0.23$ & $10.77 \pm 0.13$ & $9.50 \pm 0.18$ & $10.68 \pm 0.15$ \\
2 & $9.44 \pm 0.15$ & $10.30 \pm 0.12$ & $9.54 \pm 0.13$ & $11.40 \pm 0.16$ & $9.41 \pm 0.10$ & $10.23 \pm 0.14$ \\
3 & $11.53 \pm 0.07$ & $12.27 \pm 0.13$ & $9.32 \pm 0.09$ & $10.48 \pm 0.22$ & $12.56 \pm 0.22$ & $9.38 \pm 0.18$ \\
4 & $12.23 \pm 0.15$ & $9.29 \pm 0.12$ & $9.76 \pm 0.10$ & $10.12 \pm 0.18$ & $9.17 \pm 0.21$ & $9.29 \pm 0.26$ \\
5 & $8.56 \pm 0.29$ & $6.84 \pm 0.10$ & $6.91 \pm 0.10$ & $10.32 \pm 0.24$ & $11.54 \pm 0.28$ & $11.27 \pm 0.14$ \\
\hline
\end{tabular}

Table 3 Overall Concordance Correlation Coefficient (OCCC), Overall Accuracy (OACC), Overall Precision (OPREC) of 10 repeated ultrasound examinations to assess the cross-sectional area of the SDFT and the DDFT at three levels $(1 \mathrm{~B}, 2 \mathrm{~A}, 2 \mathrm{~B})(\mathrm{n}=5) .{ }^{*}=$ perfect agreement, ** poor agreement

\begin{tabular}{lccc}
\hline variable & OCCC & OPREC & OACC \\
\hline SDFT 1 B & 0.98 & $0.99^{*}$ & $0.99^{*}$ \\
SDFT 2A & $0.99^{*}$ & $0.99^{*}$ & $0.998^{*}$ \\
SDFT 2B & 0.98 & $0.99^{*}$ & $0.995^{*}$ \\
DDFT 1B & $0.84^{* *}$ & $0.92^{* *}$ & $0.91^{* *}$ \\
DDFT 2A & 0.98 & 0.98 & $0.99^{*}$ \\
DDFT 2B & 0.94 & 0.97 & 0.97 \\
\hline
\end{tabular}

Table 4 Mean and standard deviation (SD) of the cross-sectional area $\left(\mathrm{mm}^{2}\right)$ of 10 repeated measurements in the ultrasound images at three levels (B1, A2, B2) of the SDFT and DDFT $(n=12)$.

\begin{tabular}{ccccccc}
\hline Horse & SDFT 1B & SDFT 2A & SDFT 2B & DDFT 1B & DDFT 2A & DDFT 2B \\
\hline 1 & $8.28 \pm 0.09$ & $7.42 \pm 0.09$ & $7.68 \pm 0.06$ & $11.07 \pm 0.12$ & $9.12 \pm 0.08$ & $15.43 \pm 0.06$ \\
2 & $8.17 \pm 0.05$ & $8.16 \pm 0.16$ & $7.88 \pm 0.06$ & $11.13 \pm 0.11$ & $9.11 \pm 0.12$ & $15.31 \pm 0.13$ \\
3 & $7.10 \pm 0.09$ & $7.61 \pm 0.08$ & $7.38 \pm 0.06$ & $13.16 \pm 0.10$ & $10.25 \pm 0.09$ & $15.88 \pm 0.07$ \\
4 & $6.96 \pm 0.06$ & $7.73 \pm 0.08$ & $7.49 \pm 0.05$ & $10.68 \pm 0.05$ & $10.47 \pm 0.10$ & $15.82 \pm 0.08$ \\
5 & $8.74 \pm 0.06$ & $7.22 \pm 0.06$ & $8.07 \pm 0.06$ & $12.18 \pm 0.08$ & $9.70 \pm 0.06$ & $17.40 \pm 0.10$ \\
6 & $10.83 \pm 0.09$ & $9.89 \pm 0.05$ & $10.02 \pm 0.09$ & $9.51 \pm 0.06$ & $9.89 \pm 0.07$ & $19.24 \pm 0.03$ \\
7 & $8.06 \pm 0.05$ & $8.82 \pm 0.04$ & $8.79 \pm 0.05$ & $11.84 \pm 0.04$ & $12.16 \pm 0.03$ & $12.41 \pm 0.03$ \\
8 & $7.81 \pm 0.07$ & $7.23 \pm 0.04$ & $8.09 \pm 0.06$ & $10.82 \pm 0.06$ & $11.45 \pm 0.02$ & $13.90 \pm 0.08$ \\
9 & $7.43 \pm 0.04$ & $7.57 \pm 0.05$ & $7.15 \pm 0.04$ & $12.14 \pm 0.03$ & $10.52 \pm 0.05$ & $14.36 \pm 0.03$ \\
10 & $8.19 \pm 0.06$ & $8.19 \pm 0.06$ & $7.64 \pm 0.04$ & $10.44 \pm 0.05$ & $15.89 \pm 0.06$ & $13.88 \pm 0.05$ \\
11 & $9.09 \pm 0.05$ & $8.68 \pm 0.04$ & $8.24 \pm 0.08$ & $11.75 \pm 0.06$ & $11.18 \pm 0.05$ & $12.11 \pm 0.06$ \\
12 & $10.23 \pm 0.04$ & $8.99 \pm 0.05$ & $8.72 \pm 0.05$ & $10.57 \pm 0.06$ & $11.71 \pm 0.06$ & $15.59 \pm 0.06$ \\
\hline
\end{tabular}

Table 5 Overall Concordance Correlation Coefficient (OCCC), Overall Accuracy (OACC), Overall Precision (OPREC) of 10 repeated measurements to assess the cross-sectional area of the SDFT and the DDFT at three levels (1B, 2A, 2B) in ultrasound images ( $n=12)$. ${ }^{*}=$ perfect agreement

\begin{tabular}{lccc}
\hline variable & OCCC & OPREC & OACC \\
\hline SDFT 1B & $0.996^{*}$ & $0.997^{*}$ & $0.999^{*}$ \\
SDFT 2A & $0.99^{*}$ & $0.99^{*}$ & $0.998^{*}$ \\
SDFT 2B & $0.99^{*}$ & $0.995^{*}$ & $0.998^{*}$ \\
DDFT 1B & $0.99^{*}$ & $0.99^{*}$ & $0.998^{*}$ \\
DDFT 2A & $0.998^{*}$ & $0.998^{*}$ & $0.999^{*}$ \\
DDFT 2B & $0.998^{*}$ & $0.999^{*}$ & $0.999^{*}$ \\
\hline
\end{tabular}


mental setup showed high correlation, accuracy, and precision between the assessments of ultrasound images at the different levels of both digital flexor tendons. The OCCC between the repeat ultrasound imaging at all levels of the SDFT was very high $(\geq 0.98)$. In particular, at level $2 \mathrm{~A}$ an extremely high OCCC ( $\geq 0.99$ ) was observed. The repeat examinations of the SDFT also showed extremely high overall precision (OPREC) ( $\geq 0.99$ ) and overall accuracy (OACC) ( $\geq 0.99)$.

The OCCC for the repeat ultrasound examinations at different levels of the DDFT demonstrated a substantial or modest agreement for level $2 \mathrm{~A}(0.98)$ and $2 \mathrm{~B}(0.94)$, whereas repeat image assessment at level 1B of the DDFT showed poorer agreement (0.84) between the examinations. The OPREC and $\mathrm{OACC}$ for imaging at levels $2 \mathrm{~A}$ and $2 \mathrm{~B}$ also ranged from substantial to perfect. At level $1 \mathrm{~B}$ the values describing accuracy and precision are lower, 0.91 and 0.92 .

Image analysis - Reproducibility of ultrasound measurements of the cross-sectional area of the digital flexor tendons

Table 4 shows the mean and standard deviation of 10 measurements of the cross-sectional area $\left(\mathrm{mm}^{2}\right)$ at three different levels (B1, A2, B2) of the SDFT and DDFT in the ultrasound images of 12 horses. Very low standard deviations occur for all measurements of the cross-sectional areas of both tendons at all levels $\left(0.02-0.16 \mathrm{~mm}^{2}\right)$.

Table 5 shows all measures describing the reproducibility of repeat measurements in ultrasound images of the SDFT $(n=12)$ and the DDFT $(n=12)$ at three different levels to assess the cross-sectional areas performed by one operator. The given references and setup to analyse the ultrasound images enabled an extremely high agreement between all measurements of the cross-sectional area at all levels of both tendons (OCCC $\geq 0.99$ ). Moreover, in this study accuracy and precision were excellent (OAUC/OPREC $\geq 0.99$ ).

\section{Discussion}

Ultrasound imaging enables the monitoring of injuries or the healing progress in tendon lesions. Additionally, quantitative ultrasound measurements are performed to acquire scientific evidence of the effect of different factor - such as training, drugs, and growth - on the size of the equine flexor tendons (Gillis et al. 1995b, Kasashima et al. 2002, Avella et al. 2009, Korosue et al. 2015). In both cases the tendons' crosssectional areas of repeat examinations are measured and compared. In this context, the current study proves high agreement, precision, and accuracy of repeat ultrasound image acquisition and image analysis performed by one operator under practical circumstances. It provides valuable information that quantitative measurement of the flexor tendon's CSA is a useful option for intra-individual comparisons of this parameter under different conditions, such as disorders affecting the tendons, a changes in exercise regime, or in orthopaedic treatment.

The image acquisition in the present study showed a slightly lower OCCC, OACC, and OPREC than the image analysis. This is probably caused by the increased number of variables affecting the acquisition of ultrasound images. Even if the equipment, protocols, and operator are the same for each horse, further factors can influence examination, such as movement of the horse, environmental conditions, or slight variability in the location from which the image is obtained. For comparative examinations of the CSA at different levels, it is advisable to use clear references (accessory bone) and measuring aids (measuring stick or tape).

Furthermore, this study showed that image acquisition is more reproducible with very high OCCC, OACC, and OPREC values for the SDFT. In the study by Smith et al., higher standard deviation also occured for the CSA of the DDFT (Smith et al. 1994). This might be related to differences in the architecture, arrangement, and structure of both tendons or to the better resolution of the SDFT located closer to the probe. The images in the present study were acquired by assessing a transverse plane image of the SDFT and DDFT. The alignment of the probe was manipulated in all planes to obtain an optimal image showing the subjectively largest dimension of the cross-sectional area of each tendon at each level. Consistent perpendicular and palmar probe placement had to be ensured for each examination. Best results for both tendons simultaneously were achieved at level 2A, where anatomical conditions allow the placement of the probe to represent both structures optimally and where the SDFT and the DDFT are easier to distinguish during image analysis. Lowest values for OCCC, OACC, and OPREC occurred for the DDFT at level 1B. The differences between the levels probably occur since it might not be entirely possible to achieve the same accuracy and precision for both flexor tendons simultaneously in one image. In particular, in the distal region of the metacarpus (zone 1B), the SDFT and the DDFT show angular deviations (Bolen et al. 2007). The DDFT occurs $V$-shaped in this region and does not run parallel to the SDFT (Denoix 2005). Both flexor tendons run parallel in the middle of the metacarpal region (zone 2A) (Denoix 2005, Ross and Dyson 2011 ). Dependent on the focus on either the SDFT or the DDFT, the reproducibility might vary between both tendons. In order to increase accuracy, in the distal part it is advisable to examine both tendons separately.

All values are only valid for the given setup and protocols. With regard to this, Pickersgill stated an absence of any significant difference between different sets of equipment (Pickersgill et al. 2001). It seems that different analytical hardware and software provide a high degree of consistency and reproducibility to assess the cross-sectional area of the digital flexor tendons. However, not all available equipment provides the same technical standard and options. This study utilised commonly used ultrasound equipment with high technical standard 2,3 in addition to a standardised protocol to acquire ultrasound images at exactly the same level under the same conditions and measured the cross-sectional areas. Results may vary if the setup, technique, or protocols are changed or if the examinations are performed in different sessions. The reproducibility of image acquisition and analysis might also vary with different operators. Pickersgill et al. identified strong consistency in image acquisition through different operators without significant differences despite a considerable difference in the experience level of the operators (Pickersgill et al. 2001). This group only found significant dif- 
ferences between the two operators in image analysis to assess the CSA of the SDFT. It is advisable to use the same equipment and protocols for the duration of scientific studies and for only one operator to perform image acquisition and analysis to minimise any possible influence of these variables. During image analysis by the same operator it seems to be easier to always follow the same guidelines and to define the external border of the flexor tendons' for assessing the crosssectional area. The interpretation of the SDFT and DDFT external border location is always determined and applied by the same person, leading to similar and comparable values for this parameter for research purposes. In equine practice inter-operator or inter-technique variability for ultrasound image acquisition to assess the CSA of the flexor tendons seems to be negligible. Measurements of the CSA in the obtained images should be performed by the same operator to reduce variability in clinical follow-up examinations.

Moreover, in equine practice intervals between examinations for follow-up assessments vary and can range from weeks to months. Although this factor may rarely affect the skills and accuracy of an experienced operator, in the case of a less skilled examiner it might influence the results of repetitive evaluations of the CSA of the flexor tendons. However, no study has shown the impact of extended examination intervals on repeat measurements of the CSA of equine flexor tendons for clinical or scientific purpose.

The purpose of the study was not to examine the agreement between the values obtained with ultrasound imaging and the true sizes of the CSA at different levels. In practice and for some scientific questions, the agreement of intra-individual comparisons of the specific CSA is more important than the agreement with the true size of the tendon (Denoix 1994, Kasashima et al. 2002, Celimli et al. 2004, Avella et al. 2009). The true CSA of the flexor tendons can only be assessed by dissecting the tendons and carrying out morphometric analysis, which is practically irrelevant (Smith et al. 1994). Previously reported ultrasound measurements of SDFT and DDFT mean cross-sectional area in Warmbloods or Thoroughbreds cover a wide range of values at all levels in the tendons. Although the levels examined in this study differ slightly from those used in other studies, the mean values calculated here were considerably lower than those reported by other groups (Smith et al. 1994, Gillis et al. 1995). The size of the cross-sectional area seems to vary depending on the breed (Celimli et al. 2004, Agut et al. 2009, Boehart et al. 2010, Vilar et al. $2011)$. The inter-individual variation of the cross-sectional areas makes a direct comparison between different horses difficult (Pickersgill et al. 2001). Therefore, in order to apply ultrasound imaging for diagnostic purposes, the inter-individual comparison does not seem to be a useful approach. The values of the corresponding levels of each tendon at the contralateral limb are probably a more sensible reference, since previous studies reported no significant differences between the left and right forelimbs in the examined horses (Smith et al. 1994, Gillis et al. 1995, Pickersgill et al. 2001).

In conclusion, the current findings suggest that under field conditions with the given setup and one experienced operator, ultrasound image acquisition and analysis produce consistent results for comparing the CSA of the SDFT and the DDFT at different levels. Results may vary for the use of diffe- rent ultrasound equipment, examinations intervals and subjective skills of the operator, even if this is not proven in the literature. Except for level 1B, image acquisition enables sustainable or perfect agreement, precision, and accuracy between examinations. Image analysis with the stated software provides almost perfect agreement, precision, and accuracy between the measurements of the CSA. These results confirm hypothesis 1, except for level 1B of the DDFT. Hypotheses 2 and 3 can be confirmed. Results may vary with different equipment, examination intervals and operators. Nevertheless, the present study shows that repeat ultrasound image acquisition and analysis to assess the CSA of the equine flexor tendons in the metacarpal region performed by one experienced operator provide high agreement, precision, and accuracy. Ultrasound imaging can be stated as a useful technique for quantitative measurements of the CSA of both flexor tendons for scientific purpose or as follow-up measurements of clinical cases.

\section{Manufacturer's addresses}

1 DocCheck Ultraschall-Gel, DocCheck Medizinbedarf und Logistik GmbH, Weil im Schönbuch, Germany

2 Honda electronics HS-1200V, Honda Electronics Co., LTD., Aichi 441-3193, Japan

3 Honda electronics HLS 584M linear probe, Honda Electronics Co., LTD., Aichi 441-3193, Japan

4 ImageJ, vers. 2.0.0-rc-39/1.50b; Build: 8dcfl e65a6 Copyright 2010-2017

${ }^{5} \mathrm{R}-\mathrm{A}$ language and environment for statistical computing, R Foundation for Statistical Computing, Vienna, Austria

\section{Acknowledgements}

The authors thank Prof. Dr. C. K. W. Mülling, Dr. Charlotte von Zadow, Filip Batkowski, Lothar Kappe, Alexandra Schatz, Paul Tönnies and Elizabeth Kelly for their cooperation.

\section{Animal Welfare Statement}

This study was approved as an animal experiment by the Ethics Committee of the Thuringian state authority (Landesdirektion Thüringen, Office Langensalza) (referende number 15-003/15). Client-owned horses were included with informed consent.

\section{Funding}

Institute of Veterinary Anatomy, Faculty of Veterinary Medicine, Leipzig University and Saudi Arabian Embassy Berlin/Cultural Bureau

\section{References}

Agut A., Martinez M. L., Sanchez-Valverde M. A., Soler M. and Rodriguez M. J. (2009) Ultrasonographic characteristics (crosssectional area and relative echogenicity) of the digital flexor tendons and ligaments of the metacarpal region in Purebred Spanish horses. Vet. J. 180, 377-383 
Avella C. S., Ely E. R., Verheyen K. L. P., Price J. S., Wood J. L. N. and Smith R. K. W. (2009) Ultrasonographic assessment of the superficial digital flexor tendons of National Hunt racehorses in training over two racing seasons. Equine Vet. J. 41, 449-454

Boehart S., Arndt G., Carstanjen B. (2010) Ultrasonographic morphometric measurements of digital flexor tendons and ligaments of the palmar metacarpal region in Haflinger horses. Anat. Histol. Embryol. 39, 366-375

Bolen G., Busoni V., Jacqmot O., Snaps F. (2007) Sonographic Anatomy of the palmarodistal aspect of the equine digit. Vet. Radiol. Ultrasound 48, 270-275

Celimli N., Seyerek-Intas D., Kaya M. (2004) Morphometric measurements of flexor tendons and ligaments in Arabian horses by ultrasonographic examination and comparison with other breeds. Equine Vet. Educ. 16, 718-721

Denoix J.-M. (1994) Diagnostic techniques for identification and documentation of tendon and ligament injuries. Vet. Clin. North Am. Equine Pract. 10, 365-407

Denoix J.-M., Ed. (2005) The equine distal limb: Atlas of clinical anatomy and comparative imaging, 4th ed. Manson, London

Dowling B. A., A. J. Dart, D. R. Hodgson, R. K. W. Smith (2000) Superficial digital flexor tendonitis in the horse. Equine Vet. J. 32, 369-378

Ely E. R., Avella C. S., Price J. S., Smith R. K. W., Wood J. L. N., Verheyen K. L. P. (2009) Descriptive epidemiology of fracture, tendon and suspensory ligament injuries in National Hunt racehorses in training. Equine Vet. J. 41, 372-378

Gillis C., Meagher D. M., Cloninger A., Locatelli L., Willits N. (1995) Ultrasonographic cross-sectional area and mean echogenicity of the superficial and deep digital flexor tendons in 50 trained Thoroughbred racehorses. Am. J. Vet. Res. 56, 1265-1269

Gillis C., Sharkey N., Stover S. M., Pool, R.R., Meagher, D.M.,Willits $N$. (1995b) Effect of maturation and ageing on material and ultrasonographic properties of equine superficial digital flexor tendon. Am. J. Vet. Res. 56, 1345-1350

Hauser M. L. (1986) Ultrasonographic appearance and correlative anatomy of the soft tissues of the distal extremities in the horse. Vet. Clin. North Am. Equine Pract. 2, 127-144

Henry G. A., Patton C. S., Globe D. O. (1986) Ultrasonographic evaluation of iatrogenic injuries of the equine accessory (carpal check) ligament and superficial digital flexor tendon. Vet. Radiol. 27, 132-140

Kasashima Y., Smith R. K. W., Birch H. L., Takahashi T., Kusano K., Goodship A. E. (2002) Exercise-induced tendon hypertrophy: cross-sectional area changes during growth are influenced by exercise. Equine Vet. J. Suppl. 34, 264-268

Korosue K., Endo Y., Murase H., Ishimaru M., Nambo Y., Sato F. (2015) The cross-sectional area changes in digital flexor tendons and suspensory ligament in foals by ultrasonographic examination. Equine Vet. J. 47, 548-552

Lam K. H., Parkin T. D. H., Riggs C. M., Morgan K. L. (2007) Descriptive analysis of retirement of Thoroughbred racehorses due to tendon injuries at the Hong Kong Jockey Club (1992-2004). Equine Vet. J. 39, 143-148

Lin L. I.-K. (1989) A Concordance Correlation Coefficient to Evaluate Reproducibility. Biometrics 45, 255-268

Marr C. M., McMillan I., Boyd J. S., Wright N. G., Murray M. (1993) Ultrasonographic and histopathological findings in equine superficial digital flexor tendon injury. Equine Vet. J. 25, 23-29

McBride G. B. (2005) A proposal for strength-of-agreement criteria for Lin s concordance corelation coefficient. NIWA Clini. Report 62, 1-8

Miles C. A., Fursey G. A. J., Birch H. L., Young R. D. (1996) Factors affecting the ultrasonic properties of equine digital flexor tendons. Ultrasound in Med. \& Biol., 22, 907-915

Nicoll R. G., Wood A., Rothwell T. L. (1992) Ultrasonographical and pathological studies of equine superficial digital flexor tendons; initial observations, including tissue characterisation by analysis of image grey scale, in a thoroughbred gelding. Equine Vet. J.24, 318-320

Padaliya N. R., Ranpariya J. J., Kumar D., Javia C. B., Barvalia D. R. (2015) Ultrasonographic assessment of the equine palmar tendons. Vet. World 8, 208-212

Pickersgill C. H., Marr C. M., Reid S. W. J. (2001) Repeatability of diagnostic ultrasonography in the assessment of the equine superficial digital flexor tendon. Equine Vet. J. 33, 33-37

Ross M. W., Dyson S. J., Eds. (2011) Diagnosis and management of lameness in the horse, 2nd ed. Elsevier/Saunders, St. Louis, Mo

Smith R., Jones R., Webbon P. (1994) The cross-sectional areas of normal equine digital flexor tendons determined ultrasonographically. Equine Vet. J. 24, 460-465

Tsukiyama K., Acorda J. A., Yamada H. (1996) Evaluation of superficial digital flexor tendinitis in racing horses through gray scale histogram analysis of tendon ultrasonograms. Vet. Radiol. Ultrasound $37,46-50$

van Schie H. T. M., Bakker E. M. (2000) Structure-related echoes in ultrasonographic images of equine superficial digital flexor tendons. Am. J. Vet. Res. 61, 202-209

van Schie H. T. M., Bakker E. M., Jonker A. M., van Weeren P. R. (2000) Ultrasonographic tissue characterization of equine superficial digital flexor tendons by means of gray level statistics. Am. J. Vet. Res. 61, 210-219

van Schie J., Bakker E. M., van Weeren P. R. (1998) Ultrasonographic evaluation of equine tendons: A quantitative in vitro study of the effects of amplifier gain level, transducer-tilt, and transducerdisplacement. Vet. Radiol. Ultrasound 39, 151-160

Vilar J. M., Santana A., Espinosa J., Spinella G. (2011) Cross-sectional area of the tendons of the tarsal region in Standardbred trotter horses. Equine Vet. J. 43, 235-239

Williams R. B., Harkins L. S., Hammond C. J., Wood J. L. N. (2001) Racehorse injuries, clinical problems and fatalities recorded on British racecourses from flat racing and National Hunt racing during 1996, 1997 and 1998. Equine Vet. J. 33, 478-486

Wood A., Sehgal C. M., Polansky M. (1993) Sonographic brightness of the flexor tendons and ligaments in the metacarpal region of horses. Am. J. Vet. Res. 54, 1969-1974 